\title{
Automated Reconfigurable Antenna Impedance for Optimum Power Transfer
}

\author{
Mohammad Alibakhshikenari ${ }^{1 *}$, Bal S. Virdee ${ }^{2}$, Chan H. See ${ }^{3,4}$, Raed A. Abd-Alhameed ${ }^{5}$, Francisco Falcone ${ }^{6}$, Ernesto \\ Limiti $^{1}$ \\ ${ }^{1}$ Electronic Engineering Department, University of Rome "Tor Vergata", Via del Politecnico 1, 00133, Rome, ITALY \\ ${ }^{2}$ London Metropolitan University, Center for Communications Technology, School of Computing \& Digital Media, London N7 \\ 8DB, UK \\ ${ }^{3}$ School of Engineering \& the Built Environment, Edinburgh Napier University, 10 Colinton Road, Edinburgh, EH10 5DT, UK \\ ${ }^{4}$ School of Engineering, University of Bolton, Deane Road, Bolton, BL3 5AB, UK \\ ${ }^{5}$ Faculty of Engineering and Informatics, University of Bradford, Bradford, West Yorkshire, BD7 1DP, UK \\ ${ }^{6}$ Electric and Electronic Engineering Department, Universidad Pública de Navarra, SPAIN \\ *alibakhshikenari@ing.uniroma2.it
}

\begin{abstract}
This paper presents an approach to implement an automatically tuning antenna for optimising power transfer suitable for software defined radio (SDR). Automatic tuning is accomplished using a closed loop impedance tuning network comprising of an impedance sensor and control unit. The sensor provides the control unit with data on the transmit or receive power, and the algorithm is used to impedance of a $T$-network of $L C$ components to optimize the antenna impedance to maximise power transmission or reception. The effectiveness of the proposed tuning algorithm in relation to impedance matching and convergence on the optimum matching network goal is shown to be superior compared with the conventional tuning algorithm.
\end{abstract}

Keywords: Impedance matching, Antenna, Automatic impedance tuning, Impedance matching algorithm, power transfer, transmitter power amplifier (PA), low-noise amplifier (LNA) receiver.

\section{INTRODUCTION}

In wireless communication systems the function of an antenna is to provide an interface with free-space effective transmission and reception of the EM signals. Antennas therefore have an impact on the overall operation of the wireless system in relation to efficiency and radiation pattern [1]. In fact, the load impedance of the transmitting power amplifier (PA) is the antenna, and in the case of a receiver low-noise amplifier (LNA) the antenna is a source impedance. The feed-point impedance therefore differs widely for power amplifier efficiency or for noise performance in the case of LNAs. Furthermore, the impedance also varies with frequency and with environmental effects, for example the proximity of a portable wireless device to the user's body.

Wireless communication systems are evolving fast with ever decreasing foot-print size, expanding bandwidth and merging of wireless standards within one device. In fact, the requirement is to use a single wideband antenna that can accommodate several communications standards to facilitate software-defined operation.
Circumvention of the feed-point impedance issue requires incorporation of a reconfigurable impedance matching network interfaced between the transceiver and the antenna. In this paper an automatic antenna tuning network (AATN) is presented for integration in wireless communication systems.

\section{IMPEDANCE MATCHING AND TUNING}

The purpose of the automatic antenna tuning network, shown in Fig.1, is to optimise the transfer of power from the transmitter to the antenna and thereby maximise the signal/noise ratio of the received signal. This can be achieved by matching the impedance of the antenna $\left(Z_{A}\right)$ to the load impedance $\left(Z_{L}\right)$ over the operating frequency range [2]-[4].

AATN comprises: (i) a tuneable matching system to transform the antenna's impedance for optimum impedance matching; (ii) an impedance sensor block; and (iii) a control unit that uses the information from the sensor to modify the $L C$ values of the matching network to optimise the impedance matching to the antenna.

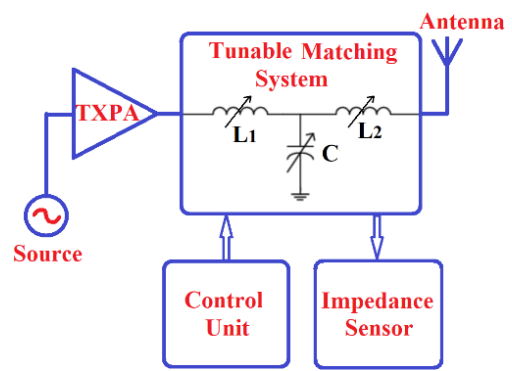

Fig.1. Block diagram of the automatic antenna tuning network with $T$ tuneable matching system.

\section{MATCHING NETWORK}

For optimum power transfer a $T$-network matching circuit in Fig. 2 is employed for impedance transformation between the antenna and (i) transmitter PA; and (ii) 
receiver LNA. This low-pass filter topology offers the advantage of minimizing noise in the system.

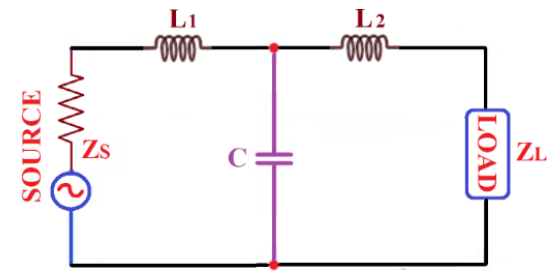

Fig.2. Schematic of the $T$-network matching circuit model.

Application of simple circuit analysis reveals the feasibility of the $T$-network to transform the source impedance to the load impedance determined by the networks $Q_{0}$ value. In the analysis it's assumed that the source impedance and load impedance are both resistive, i.e. $Z_{S}=R_{1}$ and $Z_{L}=$ $R_{2}$, so:

$$
\begin{aligned}
& X_{L 1}=R_{1} Q_{0} \\
& X_{L 2}=\frac{1}{R_{2}} \sqrt{\frac{Q_{0}+1-R_{1} R_{2}}{R_{1} R_{2}}} \\
& X_{C}=\frac{R_{1}\left(Q_{0}+1\right)}{Q_{0}+X_{L 2} R_{2}}
\end{aligned}
$$

where $X_{L 1}, X_{L 2}$, and $X_{C}$ represent the reactance of $L_{1}, L_{2}$, and $C$, respectively. Quality factor is given by:

$$
Q_{0} \geq \sqrt{\frac{R_{2}}{R_{1}}}-1
$$

In [5] the above analysis is expanded for the case where $Z_{S}$ and $Z_{L}$ are complex.

Fig. 3 shows the how the tuneable $C$ and $L$ components in the $T$-network can be implemented in practice using a switched array of fixed values. It is evident that in practice with this approach the range of impedance values for matching will be limited. Hence, a given matching network will provide a finite range of matching impedances. Therefore, antenna impedances that fall outside this range cannot be matched. In addition, the minimum incremental values of $C$ or $L$ in the network are restricted by circuit parasitics. For an on-chip application the switched series inductor array is not feasible because it would require an excessive chip footprint. In addition, the serial connection of the switches in the switch-array will lead to a high switch resistance that would contribute in additional loss.

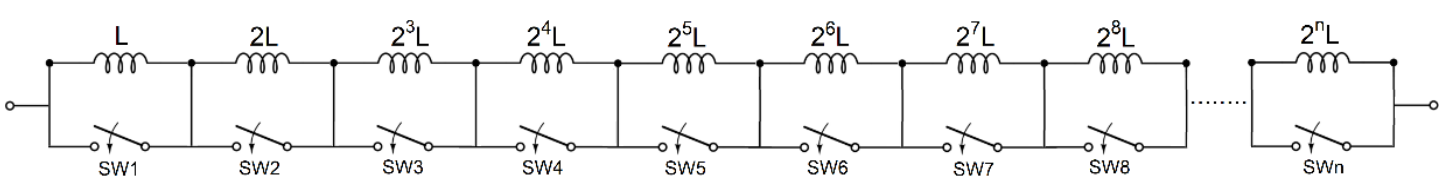

(a)

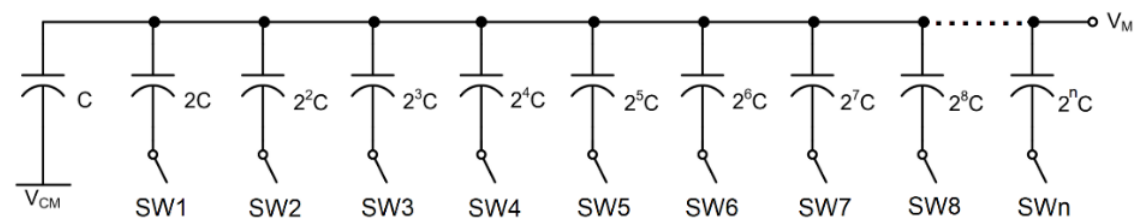

(b)

Fig.3. Switchable arrays of (a) series inductors, and (b) shunt capacitors.

In applications where bandwidth is restricted the $T$ network with series tuneable inductors (STI) can be substituted with a shunt tuneable capacitor (STC) in combination with impedance inverting networks (IINs), as illustrated in Fig.4. This topology can be applied for CMOS on-chip antenna matching, as shown in Fig.5 [6, 7], where the active device is configured as shunt switch. The proposed approach reduces the switch 'on' resistance and the generation of intermodulation products resulting from non-linearities of the switch.

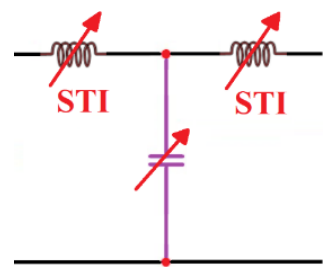

(a)

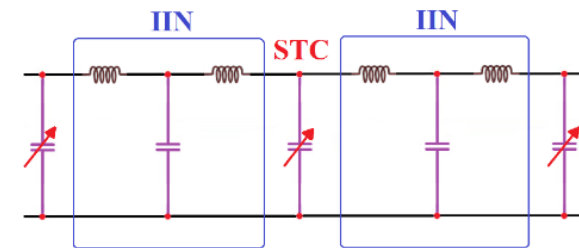

(b)

Fig.4. (a) T-network circuit model with series tuneable inductor (STI), and (b) Transformed matching network with shunt tuning capacitor (STC) in combination with impedance inverting networks (IINs).

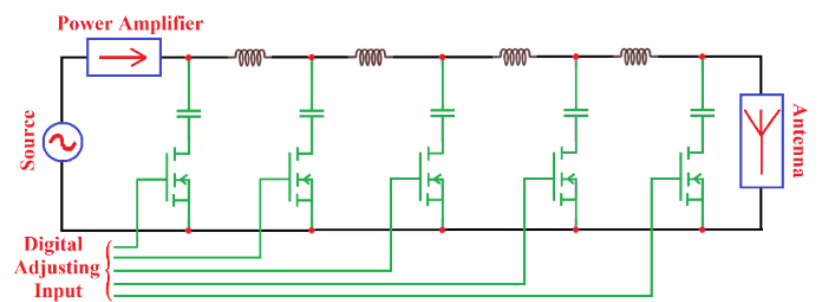

Fig.5. T-network circuit model for CMOS on-chip antenna impedance matching. 
Performance of the switches themselves can have a great impact on the impedance tuning network. In fact, the IC fabrication technology will determine the type of switches to be used. Switches implemented with active devices can introduce extra loss and circuit parasitics as well as unwanted harmonics and intermodulation products. Their limited breakdown voltages limit the power handling capacity of the matching network.

\section{IMPEDANCE SENSORS}

The purpose of the impedance sensor in Fig. 1 is to provide feedback to the tuning control unit so that the antenna's impedance is appropriately matched for optimum power transfer. The sensor essentially detects the power of the transmitted signal at the antenna terminals. The PA may operate with compromised efficiency as optimum power condition do not always correspond with the optimal load impedance. The output current and voltage from the PA can also be monitored using a phase detector. This information can be used by the control unit to modify the matching network for minimum phase differential; thus, making the load impedance at the PA output resistive for improving power transfer [8].

\section{TUNING ALGORITHM}

In a tuning network with a relatively small number of $L C$ components it may not be possible to determine suitable component values for the matching network that yields the optimum solution. However, this is not the case for a larger network. One method to obtain this is to create a look up table of matching network data for various frequencies in the operating range during an initialisation phase; the control unit then chooses the proper adjusting data from the table as the working frequency is changed. This approach obtains quick adjusting but is not able to respond to changes in the antenna impedance that happen over time without repeating the initialisation process. Here we have developed a tuning algorithm that uses impedance sensor data to iteratively converge on the optimum matching network solution. Rapid convergence is achieved because the sensor provides both signal amplitude and phase information.

To confirm the effectiveness of the proposed tuning algorithm (PTA) it is compared with the conventional tuning algorithm (CTA). In CTA as the sensors provide only amplitude information the algorithm uses a "trial-anderror" approach to determine the impedance matching mismatch. The tuning times of the algorithms was assessed over 400 simulations to determine the algorithm's speed in locating the optimal solution. For source impedance of $Z_{S}$ $=50+\mathrm{j} 20 \Omega$, load impedance of $Z_{L}=20+\mathrm{j} 30 \Omega$, source signal frequency of $5 \mathrm{GHz}$ (WiFi band), and signal amplitude of $1 \mathrm{~V}$. The optimum solution obtained gives $L_{l}$ $=5.62 \mathrm{nH}, L_{2}=3.45 \mathrm{nH}$, and $\mathrm{C}=2.18 \mathrm{pF}$. The elapsed time of PTA was $4.25 \mathrm{~s}$ and CTA was $10.54 \mathrm{~s}$. This demonstrates the effectiveness of the proposed solution.

\section{CONCLUSIONS}

In this paper an effective technique is described for automatically tuning the impedance of an antenna for integration in wireless communication systems. The proposed technique minimizes the time taken to arrive at an optimal goal by reducing the number of iterations. Compared with conventional tuning techniques the proposed algorithm rapidly converges to an optimum or close to the optimum impedance for maximum power transfer.

\section{ACKNOWLEDGMENT}

This work is partially supported by innovation programme under grant agreement H2020-MSCA-ITN2016 SECRET-722424 and the financial support from the UK Engineering and Physical Sciences Research Council (EPSRC) under grant EP/E022936/1.

\section{REFERENCES}

[1] J. D. Kraus and R. J. Marhefka, Antennas, McGraw-Hill, NY, 2002.

[2] J. R. Moritz and Y. Sun, "Frequency agile antenna tuning and matching," in Proc. Eighth International IEE Conference on HF Radio Systems and Techniques, pp. 169 -174, Surrey, UK, July 2000.

[3] Yichuang Sun, James Moritz, and Xi Zhu, "Adaptive impedance matching and antenna tuning for green software-defined and cognitive radio", 2011 IEEE 54th International Midwest Symposium on Circuits and Systems (MWSCAS), Seoul, South Korea, pp. 7-10 Aug. 2011.

[4] J. T. Aberle, B. Bakkaloglu, C. Chakrabarti, S. -H. Oh, G. A. Taylor, H. Song, A. Adhya, K. L. Melde, R. B. Whatley, and Z. Zhou, "Automatically tuning antenna for software-defined and cognitive radio," Software Defined Radio Technical Conference and Product Exposition SDR, Session 5.4, 6 pages, California, USA, Nov. 2005.

[5] Y. Sun and J. K. Fidler, "Design method for impedance matching networks", 1EE Proc. Circuits, Devices and Systems, Vol. 143, No. 4, pp 186-194, August 1996.

[6] A. Chamseddine, J. W. Haslett, and M. Okoniewski, "CMOS siliconon-sapphire tunable matching networks", EURASIP Journal on Wireless Communications and Networking, Vol. 2006, Article ID 86531, 2006.

[7] P. Sjoblom and H. Sjoland, "An adaptive impedance tuning CMOS circuit for ISM 2.4-GHz band", IEEE Transactions on Circuits and Systems-I, Vol. 52, No. 6, pp.1125 -1124, June 2005.

[8] A. Zolomy, F. Mernyei, J. Erdelyi, M. Pardoen, and G. Toth, "Automatic antenna tuning for RF transmitter IC applying high Q antenna", IEEE Radio Frequency Integrated Circuits Symposium, paper TU4D-4, pp. 501-504, June 2004. 